\title{
OR1-2
}

Oral session

\section{The effects of acute intracerebroventricular injection of chemerin-9 on systemic blood pressure in rats}

\author{
Kengo Matsumoto, Satoshi Kameshima, Kiko Hori, Muneyoshi Okada, Hideyuki Yamawaki \\ Laboratory of Veterinary Pharmacology, School of Veterinary Medicine, Kitasato University, Japan
}

Introduction: Chemerin is an adipocytokine which plays an important role in inflammatory responses and differentiation of adipocytes. We have recently clarified in mice that long-term intraperitoneal administration of chemerin significantly elevated blood pressure (BP) possibly through increased vascular structural remodeling and smooth muscle contractility. A Gi protein-coupled receptor, chemokine-like receptor 1 (CMKLR1) is one of the chemerin receptors, which is expressed in ependymal cells and peripheral sympathetic nerves in rats. The neural nuclei including rostral ventrolateral medulla in brainstem and paraventricular nucleus in hypothalamus regulate BP via the activation of sympathetic nerves. The purpose of this study was to explore the acute effects of chemerin on central regulation of BP in rats.

Materials and methods: Male Wistar rats (150-250 g, 6-7 week-old) under the control of isoflurane anesthesia were received acute administration of chemerin-9 (an active fragment of chemerin; 0.01-10 nmol) intracerebroventricularly (i. c.v.) or intravenously (i.v.). BP was invasively measured by a carotid arterial cannulation method.

Results: Systolic blood pressure (SBP) was significantly elevated by i.c.v. injection of chemerin-9 (10 nmol; $7.3 \pm 2.3$ mmHg, $n=5, p<0.05)$ compared with saline $(1.3 \pm 0.7 \mathrm{mmHg}, \mathrm{n}=5)$. While SBP was also elevated by i.v. injection of chemerin-9 (10 nmol; $2.3 \pm 0.1 \mathrm{mmHg}, \mathrm{n}=3, \mathrm{p}<0.01)$ compared with saline $(-0.1 \pm 0.3 \mathrm{mmHg}, \mathrm{n}=3)$, the hypertensive effect of chemerin-9 was weaker than that by i.c.v. injection.

Discussion: In summary, we for the first time demonstrate that chemerin mediates not only peripheral but central hypertensive effects in rats. In order to elucidate the detailed mechanisms of central hypertensive effects of chemerin, further examinations determining the changes in sympathetic nerve activity and the involvement of CMKLR1 in brain are necessary. 